\title{
Improving Photoluminescence Quantum Yield of CdTe Quantum Dots Using a Binary Solvent (Water + Glycerin) in the One-Pot Approach Synthesis
}

\author{
Lucas T. A. da Rosa, ${ }^{a}$ Isabella F. S. Aversa, ${ }^{a}$ Ellen Raphael, ${ }^{a, b}$ André S. Polo, ${ }^{\oplus c}$ \\ Alfredo Duarte, ${ }^{\circledR d}$ Marco A. Schiavon ${ }^{\circledR *, e}$ and Luciano S. Virtuoso ${ }^{a}$ \\ ${ }^{a}$ Instituto de Química, Universidade Federal de Alfenas (UNIFAL), 37130-000 Alfenas-MG, Brazil \\ ${ }^{b}$ Escola Superior de Tecnologia (EST), Universidade do Estado do Amazonas (UEA), \\ 69050-020 Manaus-AM, Brazil \\ ${ }^{c}$ Centro de Ciências Naturais e Humanas, Universidade Federal do ABC (UFABC), \\ 09210-580 Santo André-SP, Brazil \\ ${ }^{d}$ Departamento de Química Fundamental, Universidade de São Paulo (USP), \\ 05508-000 São Paulo-SP, Brazil \\ ${ }^{e}$ Departamento de Ciências Naturais, Universidade Federal de São João Del-Rei, \\ Campus Dom Bosco, Praça Dom Helvécio, 74, 36301-160 São João Del-Rei-MG, Brazil
}

\begin{abstract}
The present work describes the comparison of the optical and structural properties between CdTe quantum dots (QD) synthesized in water and in the binary solvent (water + glycerin) via one-pot approach synthesis. The optical properties of the nanocrystals obtained with different synthesis parameters were characterized by UV-visible and photoluminescence spectroscopies. The structural chracterization were performed by Fourier transform infrared spectroscopy (FTIR), zeta potential, size-distribution by dynamic light scattering (DLS), X-ray diffraction (XRD), and also by high-resolution transmission electron microscopy (HRTEM). The optical properties of CdTe QD when synthesized in the binary solvent were better, resulting in the increase of photoluminescence quantum yield $(\phi f)$. The CdTe QD prepared in $120 \mathrm{~min}$, at $\mathrm{pH}$ 10.0, in the Cd:Te molar ratio 20:1, using the molar ratio 1:1.5 of Cd:TGA (thioglycolic acid), exhibited a narrow photoluminescence band and enhanced $\phi f$ for the samples synthesized in a binary solvent in comparison to water solvent (58.4 and 49.5\%, respectively).
\end{abstract}

Keywords: CdTe, quantum dots, one-pot synthesis, synthesis conditions

\section{Introduction}

Quantum dots (QD) are nanocrystalline semiconductors that exhibit strong quantum confinement. They usually have diameters ranging from 2 to $10 \mathrm{~nm}$ and their size drastically affects the resulting optical and electronic properties, which impact strongly on their performance in devices applications. ${ }^{1-4}$

The aqueous colloidal synthesis has been an alternative to organic synthesis, that uses organometallic precursors and organic solvents, ${ }^{5,6}$ being a simpler, cheaper and less toxic to environment alternative. However, the aqueous synthesis still presents the limitation of obtained materials

*e-mail: schiavon@ufsj.edu.br with lower photoluminescence quantum yield $(\phi f)$ than compared to the ones from organic synthesis. ${ }^{7-9}$

There are several synthesis methods in aqueous medium widely used for CdTe QDs, but different strategies are being developed aiming to increase the $\phi f$, such as hydrothermal synthesis at higher temperature, ultrasonic or microwave irradiation, since usually, aqueous synthesis produces QDs with low crystallinity than the ones prepared by organometallic route, which employ high annealing temperatures..$^{10}$ Additional strategies also include variation of the precursor concentration or use of different types of surface ligands (SL), altering the $\mathrm{pH}$ of the aqueous solution, or even growth of a shell of a wide-bandgap semiconductor around the CdTe core. These different strategies are used to reduce the surface trap state density, observed as a shoulder 
in the photoluminescence (PL) spectrum in the lower energy region, which causes an increase in bandwidth, deactivates the excited state and consequently decreases the quantum yield of photoluminescence. ${ }^{11,12}$

One reported approach, ${ }^{11}$ alternatively to the conventional two-step method, is the one-pot method, that is conducted without inert gas protection and vacuum free, producing higher quality QDs, but all of them uses SL to avoid aggregation, to stabilize the colloidal dispersion and to control the size of the nanocrystals. SL have the main fuction of passivating the QDs surface, coordinating the bonds generated by decoupled orbitals of their surface, removing trap of electrons on the surface, and thereby providing an increase in the $\phi f .{ }^{13-18}$ So the SL must act as on/off dynamic behavior around the nanocrystals, sometimes allowing the nanomaterials growth (off state, disconnected from the surface) and sometimes blocking their growth (on state, bound to the surface), this behavior can be influenced by several synthesis parameters as well as the synthesis solvents choice. ${ }^{19,20}$

In this paper, we optimize some parameters in CdTe QDs one-pot aqueous synthesis, such as the $\mathrm{pH}$, concentration of SL and the molar ratio of precursors, in order to improve the optical properties. Then we changed the reaction aqueous medium by adding glycerin, a colorless and viscous liquid soluble in water, at $50 \% \mathrm{v} / \mathrm{v}$ of glycerin/water increasing by a factor of 30 the medium viscosity. ${ }^{21}$ This compound have been earlier applied as additive compound in nanocrystals synthesis, ${ }^{22}$ enabling an increase in reaction temperature, since glycerin boils at $290{ }^{\circ} \mathrm{C}$ at atmospheric pressure, leading to the change in growth kinetics of nanocrystals by promoting faster QDs formation. On the other way, it makes the liquid solution more viscous, leading to surface foam formation that prevents the agglomeration of the nanoparticles delaying the nucleation and growth rate of QDs in viscous medium. In addition, the ability of glycerin to form a complex between metal ions, as has long been reported in the literature, ${ }^{23,24}$ seems to have an important role in the mechanism of formation of QDs in glycerol-water medium. According to Stan et al. ${ }^{24}$ who studied the formation of CdSe QDs in an organic reaction medium containing glycerin, a possible complex formed between glycerin and $\mathrm{Cd}^{2+}$ at elevated temperatures can cause the slow release of cadmium cations during the nucleation and growth and thus influence the whole process kinetics, then the cadmium glycerolate complex, under certain temperatures, would be responsible for initiating the nucleation and controlling the growth stage of the particles by acting as an additional cadmium source during the synthesis of CdTe QDs. For these reasons glycerin may contribute to enhance surface properties, aiming to further improve the aqueous synthesis and the quality and luminescence properties of resulting thioglycolic acid (TGA)-capped CdTe QDs. ${ }^{25}$

\section{Experimental}

\section{Materials}

Cadmium chloride hydrate $\left(\mathrm{CdCl}_{2} \cdot \mathrm{H}_{2} \mathrm{O}, 98 \%\right)$ and sodium telluride $\left(\mathrm{Na}_{2} \mathrm{TeO}_{3}, 99 \%\right)$ were purchased from Aldrich (St. Louis, MO, USA). Sodium borohydride $\left(\mathrm{NaBH}_{4}, \geq 98 \%\right.$, Nuclear, Diadema, SP, Brazil), thioglycolic acid (TGA, 99\%, Synth, Diadema, SP, Brazil), glycerin (certified ACS grade, ProQuimios, Bangu, RJ, Brazil), isopropyl alcohol and acetone (certified ACS grade, Alphatec, São José dos Pinhais, PR, Brazil) were used without purification.

\section{CdTe QD synthesis}

CdTe QDs were synthesized in aqueous medium using the one-pot approach previously reported. ${ }^{26}$ In brief, $0.4 \mathrm{mmol}$ of $\mathrm{CdCl}_{2} \cdot \mathrm{H}_{2} \mathrm{O}$ and $0.02 \mathrm{mmol}$ of $\mathrm{Na}_{2} \mathrm{TeO}_{3}$ were dissolved in $80 \mathrm{~mL}$ of water and the $\mathrm{pH}$ was adjusted to 10.0. To this solution, $0.1 \mathrm{mmol}$ of sodium borohydride (reducing agent) and TGA (SL) in a different molar ratios were used. The reaction was heated to reflux $\left(98^{\circ} \mathrm{C}\right)$ for $120 \mathrm{~min}$. For samples prepared in water/glycerin mixture, the $80 \mathrm{~mL}$ of water was replaced by the binary solvent prepared by the mixture of $40 \mathrm{~mL}$ of water and $40 \mathrm{~mL}$ of glycerin and was heated to reflux $\left(104{ }^{\circ} \mathrm{C}\right)$ for $120 \mathrm{~min}$. The as-synthesized CdTe QDs were then precipitated with $160 \mathrm{~mL}$ of acetone for synthesis in water and isopropyl alcohol for synthesis in water + glycerin. QDs were stored under refrigeration until further characterization. ${ }^{27}$

\section{Characterization}

UV-visible absorption spectra were collected in 300-700 nm range using a Thermo Scientific ${ }^{\mathrm{TM}}$ Evolution 60S UV-Visible spectrophotometer. PL spectra were collected in 400-700 nm range recorded using a Cary Eclipse (Varian) spectrometer. For PL decay time measurements, the photoluminescence from the samples were collected by a PicoQuant Fluotime 300 spectrophotometer, using a diode laser as excitation source at $375 \mathrm{~nm}$ (LDH-P-C-375B, $40 \mathrm{MHz}$ and $52 \mathrm{ps}$ pulse) controlled by a driver (PDL-820) as described elsewhere. ${ }^{28}$ The photoluminescence quantum yield $(\phi f)$ of CdTe QDs was determined by using Rhodamine 6G $(\phi f=95 \%)$ as PL reference. ${ }^{29}$ High-resolution transmission electron microscope 
(HRTEM) images were collected on a JEOL JEM-2100 operated at $200 \mathrm{kV}$. Samples for HRTEM studies were prepared by dropping diluted aqueous solution of $\mathrm{CdTe}$ QDs previously stabilized by BSA (bovine serum albumin, 0.05 wt.\%) onto 400 mesh carbon-coated (3-4 nm) copper grids.

Dynamic light scattering (DLS) and zeta potential measurements were carried out using a Zetasizer Nano $\mathrm{ZS}$, from Malvern, with $\mathrm{He}-\mathrm{Ne}$ laser at $633 \mathrm{~nm}$ and power of $4 \mathrm{~mW}$. The $\mathrm{pH}$ dependence of the zeta potential of the CdTe QDs sample was measured using a Malvern Zetasizer Nano-ZS with an MPT-2 automatic titrator. The $\mathrm{pH}$ adjustments were performed across a $\mathrm{pH}$ range of 3 to 12 using $0.5 \mathrm{pH}$ unit increments. Samples were precipitated by isopropyl alcohol and acetone and dried under vacuum for X-ray powder diffraction (XRD) and Fourier transform infrared spectroscopy (FTIR) characterizations. XRD patterns were recorded on a Rigaku Ultima IV X-ray diffractometer over $2 \theta$ range of $10-80^{\circ}$ with step scan $0.01^{\circ}$ and employing $\mathrm{Cu} \mathrm{K} \alpha$ radiation $(\lambda=1.5406 \AA)$. FTIR spectra were recorded on a Nicoleti S50 FTIR coupled to Pike Gladi ATR Technologies (Thermo Scientific, USA).

\section{Results and Discussion}

\section{Optical properties of the QDs prepared}

The synthesis of the CdTe QDs was first conducted in water in order to establish the synthetic parameters. The reaction time and the amount of the surface stabilizer was changed systematically. Once established the best conditions of synthesis in the water medium, these conditions were fixed and the synthesis was repeated in water + glycerin solvent.

The nanocrystals formed in water or water + glycerin media show a well-resolved excitonic absorption peak, which is red shifted as the reaction time increases due to the growth of the particle size. The nanocrystals mean diameter (D) was first estimated according to the empirical formula from Peng and co-workers ${ }^{30}$ (equation 1) and are listed in Table 1, along to other spectroscopic parameters.

$\mathrm{D}=\left(9.8127 \times 10^{-7}\right) \lambda^{3}-\left(1.7147 \times 10^{-3}\right) \lambda^{2}+$

$(1.0064) \lambda-194.84$

where $\lambda$ is the first maximum absorption.

Synthesized CdTe QD exhibits broad absorption and emission bands, which are ascribed to the contribution of surface or core defect states of the QDs and the size distribution as well. The low energy emission are related to surface traps, while the higher energy emission are ascribed to the core of the QD, which is the main excitonic band. ${ }^{31}$ Figure 1 shows sets of emission and absorption spectra acquired for some CdTe QDs samples prepared (data extracted are in Table 1) and as the time reaction increases, the PL emission and UV-visible absorption peaks are redshifted, due to increase of the QD sizes.

The Stokes shifts were calculated from the difference between positions of the band maxima of the absorption and emission spectra of the same electronic transition. The emission from samples with Stokes shift values of large magnitude has greater contribution of the surface emission band and can also be correlated with the $\phi f$. In general, samples with higher $\phi f$ presented lower Stokes shift values. ${ }^{32}$ It is also possible to assess the particle size distribution by determining the full width at half maximum (FWHM) of the emission band, when this band is symmetrical it indicates small contribution from surface traps defects. This parameter considers that with larger FWHM, more polydisperse is the size distribution of the nanocrystals formed. The $\phi f$ was also determined for the different synthetic conditions at the times of $30,60,90$

Table 1. Optical parameters of CdTe QDs synthesized in water and water + glycerin for 120 min, using TGA at different Cd:TGA molar ratios in pH 10 and Cd:Te molar ratio of 20:1

\begin{tabular}{|c|c|c|c|c|c|c|}
\hline QD & $\begin{array}{c}\text { Cd:TGA } \\
\text { molar ratio }\end{array}$ & Solvent & Size / nm & $\phi f / \%$ & Stokes shift / nm & FWHM / nm \\
\hline 1 & $1: 1.5$ & $\mathrm{~W}$ & 2.53 & 49.5 & 34.19 & 51.07 \\
\hline 2 & $1: 2$ & $\mathrm{~W}$ & 1.73 & 13.1 & 55.32 & - \\
\hline 3 & $1: 2.5$ & W & 1.31 & 5.3 & 63.83 & - \\
\hline 4 & $1: 3$ & W & - & 1.3 & 99.66 & - \\
\hline 5 & $1: 1.5$ & $\mathrm{~W}+\mathrm{G}$ & 2.48 & 58.4 & 33.58 & 41.69 \\
\hline 6 & $1: 2$ & $\mathrm{~W}+\mathrm{G}$ & 2.13 & 23.7 & 38.92 & - \\
\hline 7 & $1: 2.5$ & $\mathrm{~W}+\mathrm{G}$ & 1.87 & 4.4 & 54.57 & - \\
\hline 8 & $1: 3$ & $\mathrm{~W}+\mathrm{G}$ & - & - & - & - \\
\hline
\end{tabular}

QD: quantum dots; TGA: thioglycolic acid; $\phi f$ : photoluminescence quantum yield; FWHM: full width at half maximum; W: water; G: glycerin. 

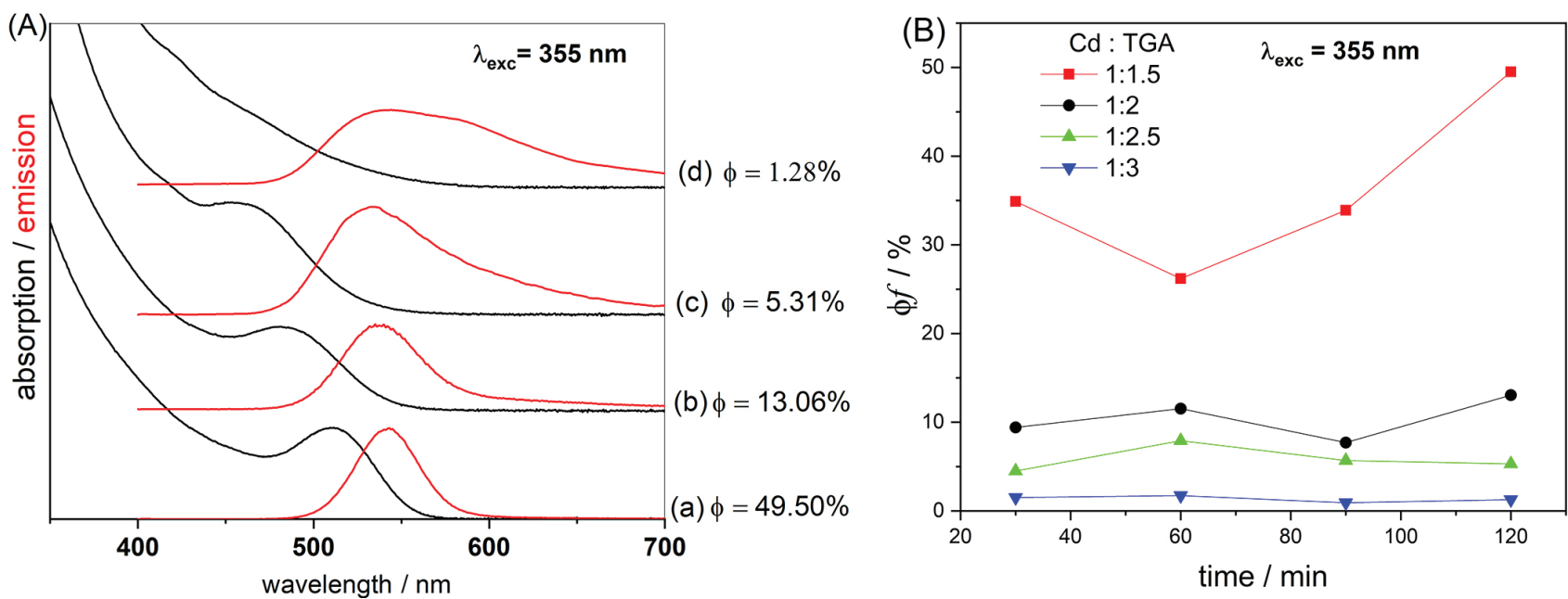

Figure 1. (A) Absorption and emission spectra of CdTe QDs synthesized at $120 \mathrm{~min}$ in water with different Cd:TGA ratios = 1:1.5 (a), 1:2 (b), 1:2.5 (c) and 1:3 (d); (B) $\phi f$ of CdTe QDs at different reaction times in water.

and 120 min of reaction, using Rhodamine $6 \mathrm{G}(\phi f=95 \%)$ as a reference. The synthesis parameters for each QDs synthesized with their estimated mean diameter, $\phi f$, Stokes shift and FWHM are summarized in Table 1.

Figure 1A shows the absorption and emission spectra for CdTe QDs synthesized at $120 \mathrm{~min}$ in water, with molar ratio 20:1 of $\mathrm{Cd}: \mathrm{Te}$, at $\mathrm{pH} 10$, and with different molar ratio of Cd:TGA. Synthetic procedure using longer times than 120 min were also investigated, and they exhibited worst results and are not shown. In the spectra, it can be observed that besides the main excitonic emission peak, ${ }^{31}$ low-energy emission tails, ascribed to emission from energy levels of the surface traps, become more prominent for the CdTe QDs prepared in lower TGA concentration. This behavior indicates that TGA promotes stabilization of the emission by suppressing the surface trap states.

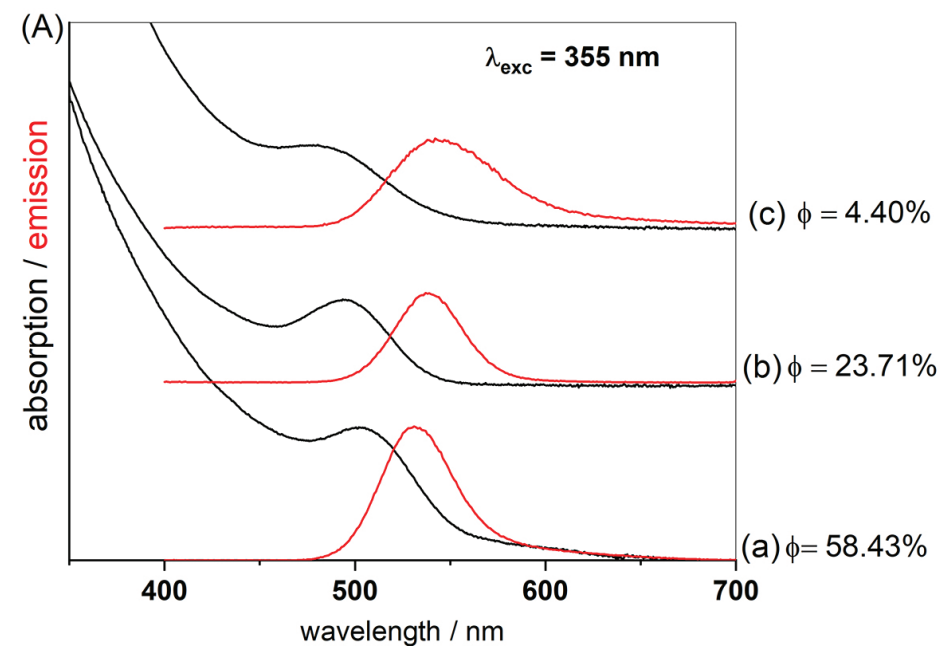

The analyses revealed that the synthesis in the condition of $\mathrm{pH}$ 10.0, Cd:Te molar ratio of 20:1 and using SL in molar ratio of $\mathrm{Cd}$ :TGA $=1: 1.5$, resulted in QDs having a more homogeneous size distribution (with lower Stokes shift value), and $\phi f$ of $49.5 \%$. It was also possible to observe an increase in $\phi f$ from samples prepared by reacting for 30 to 120 min (Figure 1B), indicating that the growth of the nanocrystals promotes a decrease in the trap bands leading to an improvement in optical quality of the obtained QDs. ${ }^{26,27,33}$

The best conditions for the CdTe QDs synthesis in aqueous medium were employed for a comparative study replacing water by a binary solvent composed of water + glycerin. The absorption and emission spectra of CdTe QD synthesized in binary solvent water + glycerin and the $\phi f$ at different reaction times are shown in Figures 2A and $2 \mathrm{~B}$, respectively.

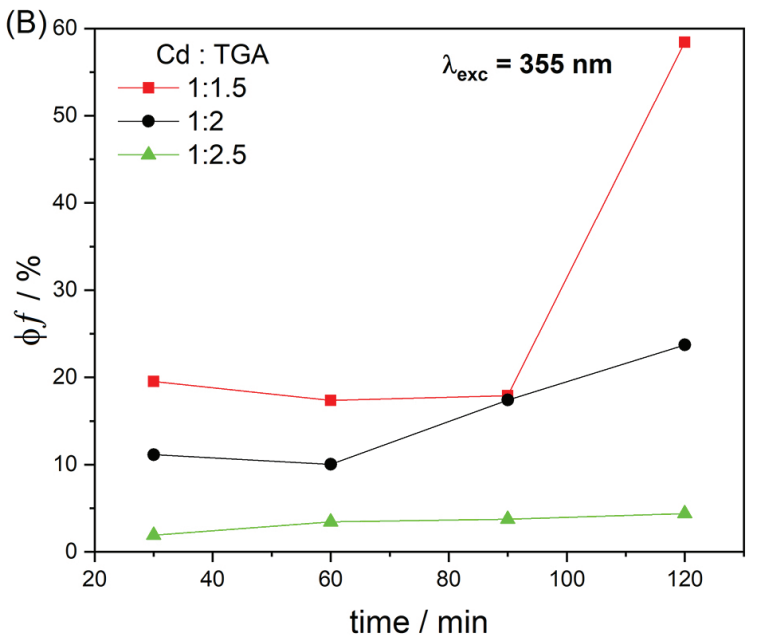

Figure 2. (A) Absorption and emission spectra of CdTe QDs synthesized at $120 \mathrm{~min}$ in binary solvent (water + glycerin) with different Cd:TGA ratios = 1:1.5 (a), 1:2 (b) and 1:2.5 (c); (B) $\phi f$ of CdTe QDs at different reaction times in water + glycerin. 
The surface quality of QDs is crucial for their optical quality, since the surface traps play an important role on the emission characteristic of these samples. Considering that in a smaller material size there is an increase in the surface/core ratio, the contribution of the surface traps emission becomes more significant as the size is reduced. Surface control usually occurs by using surface ligands that act to reduce surface defects by controlling the particle growth kinetics.

From the photoluminescence spectra of the samples showed in Figure 3 it can be observed an increase of the emission intensity due to the presence of glycerin as well as the molar ratio of Cd:TGA. It is important to notice that the spectra are mainly characterized by just one peak with a maximum around $550 \mathrm{~nm}$ for all samples, which can be assigned to an emission exclusively from quantized core. ${ }^{34}$

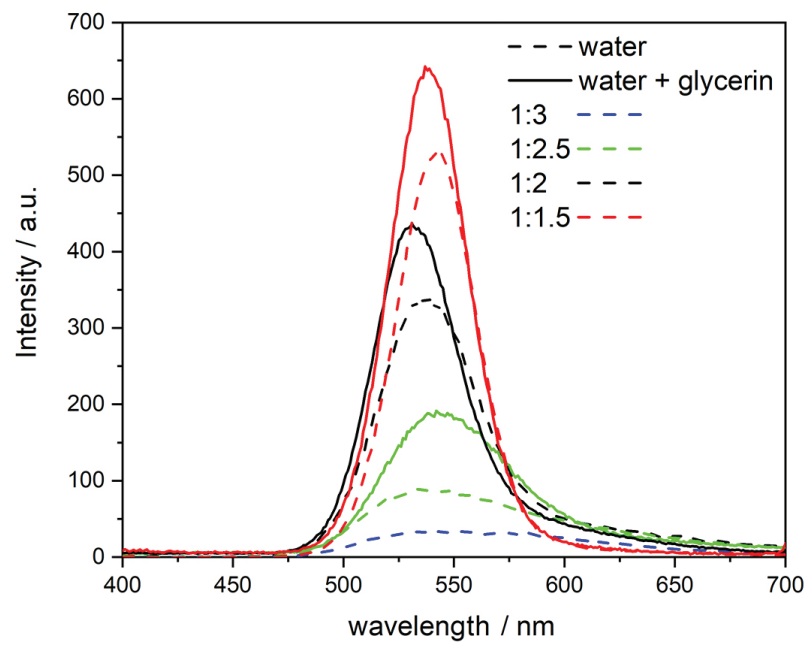

Figure 3. PL spectra of CdTe QD synthesized at reaction time of $120 \mathrm{~min}$, in water (dashed line) and water + glycerin (continuous line), with different Cd:TGA molar ratios: 1:1.5, 1:2, 1:2.5 and 1:3.

TGA was used as SL, but probably glycerin may also act as ligand of the surface on synthesized QDs. It can also be observed in Figure 3 that the spectrum obtained with water + glycerin shows the main excitonic peak slightly blue shifted in comparison with the ones registered in water. This effect may be due the refraction index of this mixture, but may also be assigned to the presence of glycerin acting as SL on the QDs, which causes an increase in the contribution of the core excitonic in comparison to the spectra of samples with only water, suggesting a reduction of surface traps. It is notorious that the presence of glycerin in the reaction medium increased the luminescence of the QD obtained under the same conditions of synthesis, in comparison with those performed using only water as solvent. This increase in the luminescence may be then ascribed to the reduction of trap states increasing the intensity of photoluminescence of the QDs.

By comparing two QDs prepared with Cd:TGA molar ratio of $1: 1.5$ with the same synthesis time $(120 \mathrm{~min})$ but in the different solvents studied here: water and water + glycerin, the $\phi f$ obtained were 49.5 and $58.4 \%$, respectively (see Table 1). This difference in the values of $\phi f$ is a strong evidence that the presence of glycerin actually improves the $\phi f$, probably by decreasing the traps on the QDs surface. A proper correlation between the values of $\phi f$ and Stokes shift was proposed by Rogach et al..$^{32}$ as a rapid technique to evaluate the quality of the samples, without involving the comparison with luminescence standards. According to this proposal, high $\phi f$ samples generally exhibit a lower Stokes shift than low $\phi f$ samples. As we can see in data from Table 1, this fact was observed for samples with higher $\phi f$, which were prepared using the binary solvent.

The PL lifetimes of selected samples were also monitored, as can be seen at Figure 4 by the PL decay profiles. The PL lifetimes of core states were probed at the maximum wavelength observed in the emission spectra (around $530 \mathrm{~nm}$ ). The values of each curve are presented in Table 2.

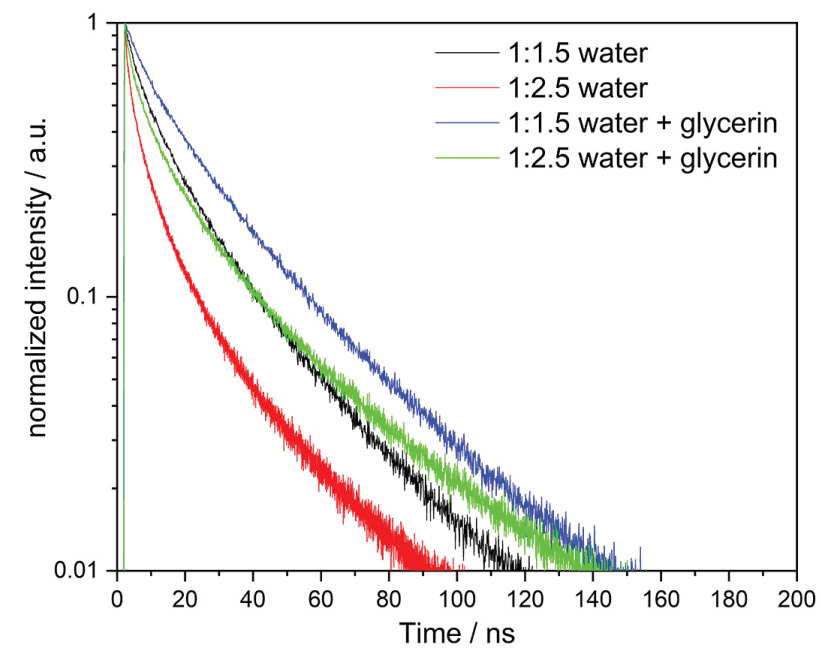

Figure 4. PL decay profiles of quantized states (probe $=530 \mathrm{~nm}$ ) for $\mathrm{CdTe}$ QDs obtained with $120 \mathrm{~min}$ of synthesis.

Table 2. PL emission decay parameters determined for selected samples (obtained with synthesis time of $120 \mathrm{~min}$ ) in both solvents studied

\begin{tabular}{lccc}
\hline $\begin{array}{l}\text { Cd:TGA molar } \\
\text { ratio }\end{array}$ & Solvent & $\tau 1$ & $\tau 2$ \\
\hline $1: 1.5$ & $\mathrm{~W}$ & $6.0(53 \%)$ & $23.5(47 \%)$ \\
$1: 1.5$ & $\mathrm{~W}+\mathrm{G}$ & $6.8(43 \%)$ & $28(57 \%)$ \\
$1: 2.5$ & $\mathrm{~W}$ & $3.44(73 \%)$ & $18.3(27 \%)$ \\
$1: 2.5$ & $\mathrm{~W}+\mathrm{G}$ & $5.27(61 \%)$ & $26.8(39 \%)$ \\
\hline
\end{tabular}

TGA: thioglycolic acid; $\tau 1$ : decay time 1 ; $\tau 2$ : decay time 2 ; W: water; G: glycerin. 
The emission lifetime is composed by two components. The energy levels for CdTe quantum dots are composed by $5 p$ orbitals, doubly degenerated, from Te on the top of valence band and 5 s orbitals of $\mathrm{Cd}$, nondegenerated, on the bottom of the conduction band. Due to the relatively large spin-orbit coupling of these orbitals, they are expected to mix weakly with each other. Consequently, electron-hole exchange interactions in these QDs split the manifold into higher energy triplet states and lower energy quintuplet states. The existence of a biexponential emission decay is proposed to occur from the superposition of the triplet and quintuplet energy levels and it exhibits the shorter lifetimes. The second and longer emission lifetimes are from the triplet-quintuplet exciton equilibrium. ${ }^{33}$

As can be seen in Table 2, longer decay times were obtained for the samples obtained from the synthesis with the binary solvent (water + glycerin), which also indicates that these samples have less surface defects. The higher $\phi f$ for these samples are also in accordance with this observation. In addition, the lifetimes decay values are in agreement with the ones observed for other CdTe samples, already published in literature. . $^{35,36}$

From the optical properties of the samples studied we can conclude that the samples prepared in the binary solvent have better properties than the ones synthesized just in water, maily due to the effect of glycerin in the synthesis, and also with the beneficial effect of this molecule as SL on the surface of CdTe QDs.

\section{Structural and morphological characterization of QDs}

The crystal structure of the QDs prepared were evaluated by powder XRD pattern as can be seen in Figure 5. The patterns obtained are similar and typical of crystalline nanoparticles, which have few planes to difract. The patterns exhibits broad peaks at $2 \theta=25.01,41.85$

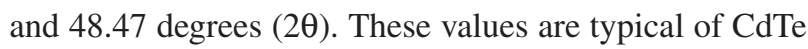
QDs, and correspond to Miller indices (111), (220) and (311), ascribed to lattice planes of a cubic structure (zinc blende) CdTe (JCPDS-75-0106). ${ }^{37-39}$ There is no evidence of other phases.

The FTIR spectra of TGA and of the QDs prepared in water and water + glycerin mixture are shown in Figure 6, and the main absorptions of the molecular groups of SL are displayed in Table 3. The most pronounced IR absorption bands occur at $3500-3000 \mathrm{~cm}^{-1}\left(v_{\mathrm{OH}}\right), 2926 \mathrm{~cm}^{-1}\left(v_{\mathrm{CH} 2}\right)$, $2574 \mathrm{~cm}^{-1}\left(v_{\mathrm{SH}}\right), 1707 \mathrm{~cm}^{-1}\left(v_{\mathrm{C}=0}\right), 1222 \mathrm{~cm}^{-1}\left(v_{\mathrm{C}-\mathrm{O}}\right)$. For the bound ligands in the CdTe samples, the $\mathrm{COO}^{-}$vibrations at 1562 and $1397 \mathrm{~cm}^{-1}$ are consistent with the fact that during the synthesis with the high value of $\mathrm{pH}$ (ca. 10), the carboxylic acid group is deprotonated, since its $\mathrm{p} K_{\mathrm{COOH}}$ value

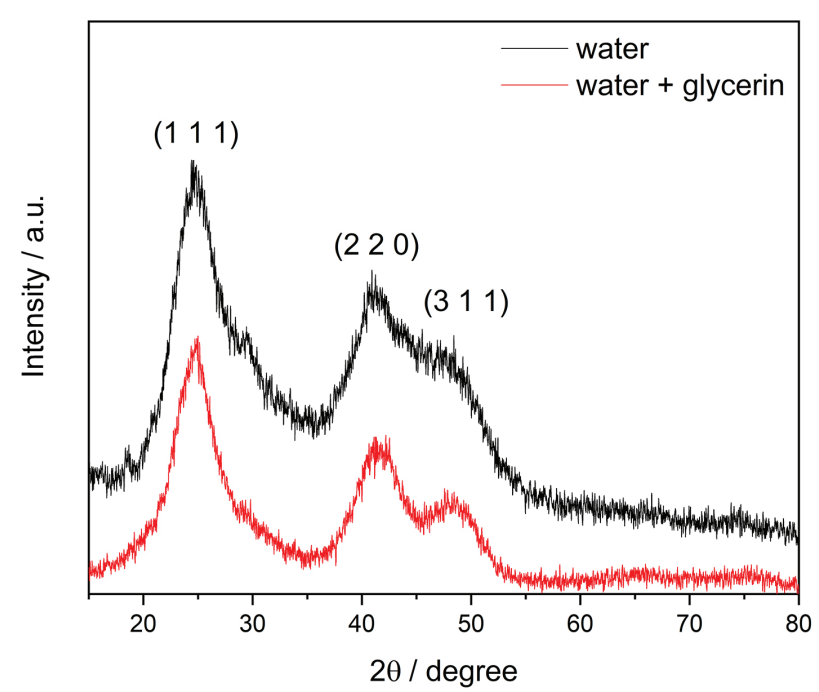

Figure 5. XRD patterns of CdTe QDs prepared in water and water + glycerin, obtained at $120 \mathrm{~min}$ of synthesis.

is 3.67. The $\mathrm{S}-\mathrm{H}$ vibrations $\left(2574 \mathrm{~cm}^{-1}\right)$ are not detectable in the IR spectra of any of the bound ligands, which is expected for thiols covalently bound to the surface of nanocrystals, ${ }^{40-42}$ or by the complete deprotonation of S-H group $\left(\mathrm{p} K_{\mathrm{a}}=8.3\right)$. This technique is not suitable to detect the presence of glycerin molecules that may be possibly attached to the surface of nanocrystals, since their amount should be low, and the presence of same functional groups are presented in TGA SL molecule, such as $\mathrm{C}-\mathrm{H}$ and $\mathrm{C}-\mathrm{O}-\mathrm{H}$. In addition, the presence of glycerin may not modify the interaction of the thiol-ligand with the CdTe QD, since this bound should be stronger due to the nature of the functional groups and the $\mathrm{Cd}$ and $\mathrm{Te}$ atoms from the semiconductor surface.

It is well-known in the QDs literature ${ }^{26}$ that the carboxylic acid acts as an electrostatic stabilizer of the QDs

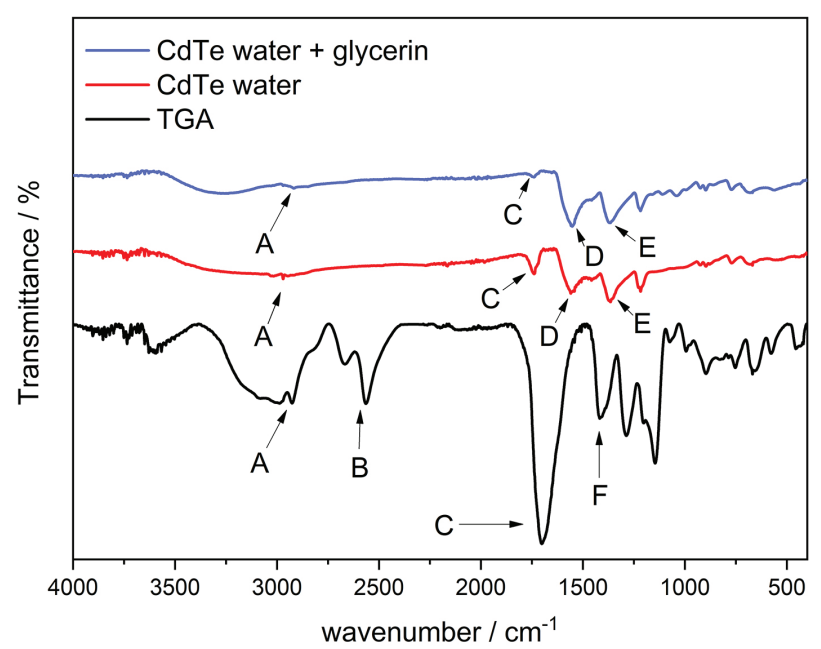

Figure 6. FTIR spectra registered with ATR (attenuated total reflectance) of TGA and CdTe QDs prepared in water and water + glycerin obtained at $120 \mathrm{~min}$ of synthesis. 
Table 3. Assigned vibrations from the molecular groups of the functional groups from TGA and the CdTe stabilized with TGA

\begin{tabular}{lcc}
\hline Assigned vibration & $\begin{array}{c}\text { Wavenumber } \\
\text { observed } / \mathrm{cm}^{-1}\end{array}$ & $\begin{array}{c}\text { Symbol in the } \\
\text { spectra }\end{array}$ \\
\hline$v_{\mathrm{CH} 2}$ & 2926 & $\mathrm{~A}$ \\
$v_{\mathrm{SH}}$ & 2574 & $\mathrm{~B}$ \\
$v_{\mathrm{C}=\mathrm{O}}$ & 1707 & $\mathrm{C}$ \\
$v_{\mathrm{C}=\mathrm{O}}$ (carboxylate) & 1562 & $\mathrm{D}$ \\
$v_{\mathrm{C}=\mathrm{O}}$ (carboxylate) & 1397 & $\mathrm{E}$ \\
$v_{\mathrm{C}-\mathrm{O}}$ & 1222 & $\mathrm{~F}$ \\
\hline
\end{tabular}

when in solution, while the thiol group has a chemical bond to the nanoparticle. The binding of $\mathrm{CdTe}-\mathrm{S}-\mathrm{R}$ has poor interaction which leads to the formation of trap bands in the material, depending on one more type of stabilization to decrease this effect. ${ }^{24}$ FTIR exhibits the disappearance of the $\mathrm{S}-\mathrm{H}$ band in both CdTe samples spectra, due to the formation of the chemical bond of the sulfur to the QD, ${ }^{19}$ while it is also possible to observe qualitatively in the spectra a relative decrease in $\mathrm{C}=\mathrm{O}$ band intensity in $\mathrm{CdTe}$ QDs synthesized in water + glycerin, indicating that the glycerin may be acting as an additional surface ligand. ${ }^{24}$

In order to get more evidences of the presence of glycerin as SL together with TGA on CdTe QDs, zeta potential measurements of the QD prepared in water and water + glycerin was carried out in buffer solutions at different $\mathrm{pHs}$, in a range between 3 and 12, as shown in Figure 7.

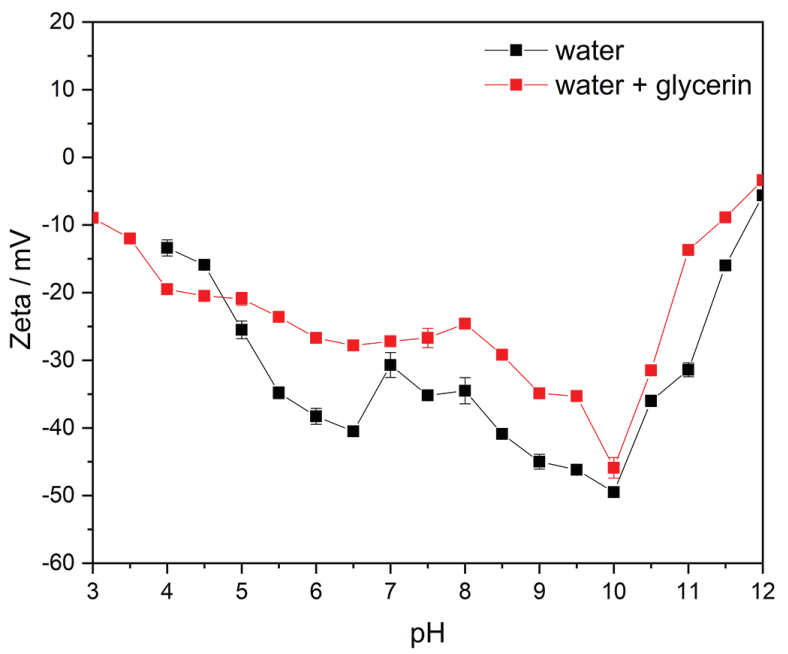

Figure 7. Zeta potential values as function of the $\mathrm{pH}$ for CdTe samples synthesized in water and water + glycerin, obtained at $120 \mathrm{~min}$ of synthesis.

As can be seen in the graph displayed in Figure 7, the zeta potential profile as function of $\mathrm{pH}$ for both samples are similar in the studied $\mathrm{pH}$ range. When the value of the zeta potential is greater than $|30 \mathrm{mV}|$, the charges in the vicinity of the particles repel each other avoiding agglomeration, providing colloidal stability. ${ }^{43}$ Therefore both samples showed relatively good colloidal stability in the $\mathrm{pH}$ range. Experimental values of zeta potential ranging from $-13.4 \mathrm{mV}$ at $\mathrm{pH} 4$ to $-40.5 \mathrm{mV}$ at $\mathrm{pH} 6.5$ and $-49.5 \mathrm{mV}$ at $\mathrm{pH}$ 10 and reaching values of $-5.63 \mathrm{mV}$ at $\mathrm{pH} 12$ were observed for the QDs prepared in water. Although the overall profile is similar, it can be observed that the variation of the zeta potential values was lower: $-8.85 \mathrm{mV}$ at $\mathrm{pH} 3,-45.9 \mathrm{mV}$ at $\mathrm{pH} 10$ and reaching values of $-3.39 \mathrm{mV}$ at $\mathrm{pH} 12$ for the $\mathrm{CdTe}$ synthesized in the binary solvent mixture. In $\mathrm{pH}$ below 4 the measurements of zeta potential could not be performed due to precipitation of the material in solution, probably due to the increase in the zeta potential value (approaching to $0 \mathrm{mV}$ ) leading to the formation of agglomerates. By contrary, on $\mathrm{pH}$ values higher than 10 the zeta potential values increase due to aggregation of the nanoparticles. The best values of zeta potential for both samples, were at $\mathrm{pH} 10$, which is the same used in the synthesis media.

The most important observation here is that CdTe QDs prepared in water have lower zeta potential values in the $\mathrm{pH}$ range from 4-10, when compared with zeta potential values to those prepared in water + glycerin mixture. This may also support the presence of some glycerin molecules acting as SL on the CdTe nanocrystals, which could cause the loss of some TGA, due to steric hindrance, and thus, decrease the zeta potential for these samples. This fact is in agreement with the optical properties for this sample, and with the DLS results presented below, in which the hydrodynamic radius was bigger than the one obtained for CdTe synthesized in water.

The size distribution of the synthesized CdTe was then measured by DLS, as can be seen in Figure 8. The obtained mean values were $2.59 \pm 0.02$ and $3.40 \pm 0.04 \mathrm{~nm}$ for the

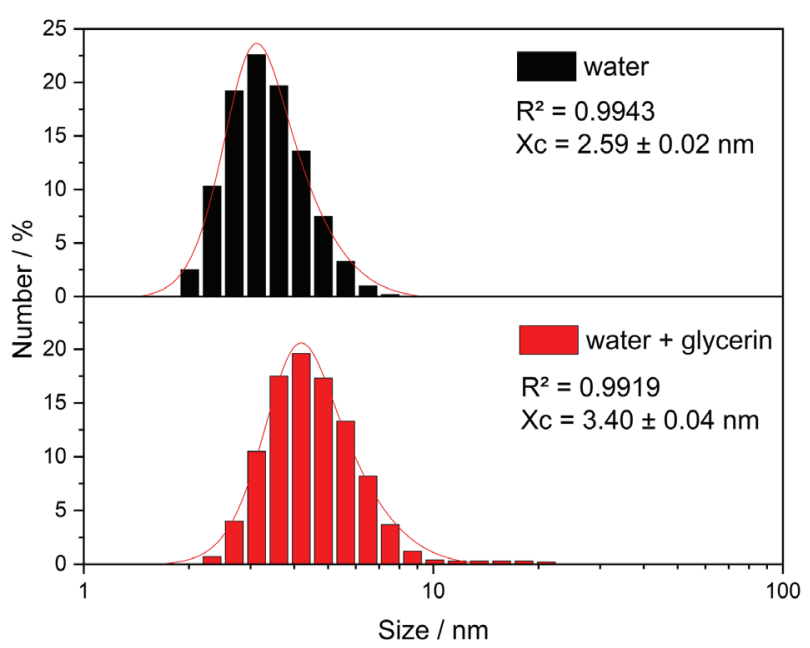

Figure 8. Hydrodynamic size distribution of CdTe QD prepared with $120 \mathrm{~min}$ of synthesis in water and water + glycerin. 

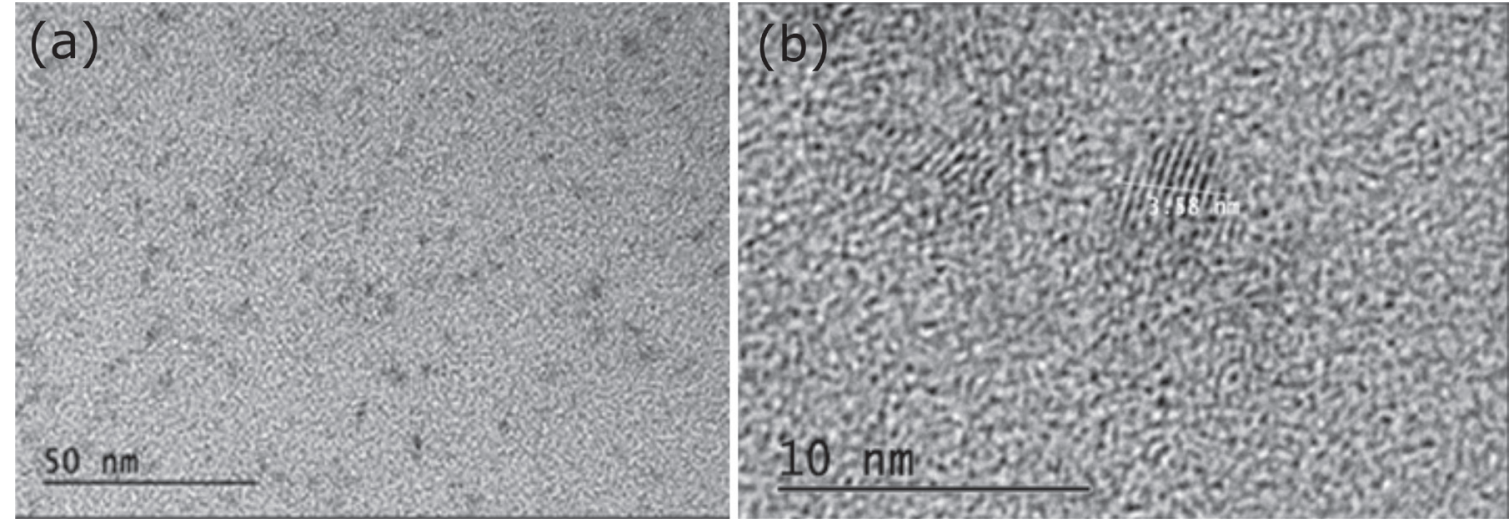

Figure 9. TEM images from sample synthesized with Cd:TGA molar ratio of 1:1.5, pH 10 and refluxed for 120 min in water. The micrographs show different magnification scales: (a) 50 and (b) $10 \mathrm{~nm}$.

synthesis in water and water + glycerin, respectively. The value obtained for the synthesis in water is close to that estimated by the Peng equation $(2.53 \mathrm{~nm})$ and to other results reported in the literature. ${ }^{44}$

Although the absorption and emission spectra of the two syntheses are similar, the average size measured for the synthesis in water + glycerin is different from the one prepared in water. This difference may be explained by the presence of the glycerin as SL, since this technique provides the hydrodynamic radius. ${ }^{45}$

Finally, in Figures $9 \mathrm{a}$ and $9 \mathrm{~b}$ are displayed representative TEM micrographs for a sample obtained after $120 \mathrm{~min}$ of synthesis in aqueous medium. In Figure 9a it is observed that the colloid comprises particles almost spherical in shape. The interplanar distance ( $3.58 \AA$ ) extracted from electron diffraction pattern observed in Figure $9 \mathrm{~b}$ can be attributed to (111) direction, also in agreement with XRD data for CdTe-QDs face-centered cubic phases, as previously reported. ${ }^{46,47}$ Similar images were acquired for the nanoparticles obtained in the binary system (not shown).

\section{Conclusions}

Water-soluble CdTe QDs have been successfully synthesized both in water and in a binary solvent prepared by water + glycerin, in the same volume proportions, using the thioglycolic acid as surface ligand. The best molar ratio for Cd:TGA was 1:1.5. The synthesis, using water as solvent, was performed at $\mathrm{pH} 10.0$, in the $20: 1 \mathrm{Cd}$ :Te ratio, showing the smallest distribution of nanocrystals and good optical properties, such as low Stokes shift and $\phi f$ of $49.5 \%$.

The results indicate that the syntheses performed under the same conditions, but using binary solvent, presented better parameters, mainly in relation to the photoluminescence quantum yield, which reached up to $58.43 \%$. The zeta potential values strongly suggest that glycerin may be present on the surface of the material, and the decrease in the relative intensity of $-\mathrm{COOH}$ band observed in FTIR corroborates this proposal.

We have observed that the presence of glycerin in the reaction media favored the obtention of CdTe with better optical properties than the ones synthesized just in water, by reducing the surface defects, and acting also as SL together with TGA. These results may suggest that glycerin could be used in aqueous synthesis to get better optical properties of aqueous synthesized CdTe.

\section{Acknowledgments}

This work was supported by FAPEMIG (Fundação de Amparo à Pesquisa do Estado de Minas Gerais), CNPq (Conselho Nacional de Desenvolvimento Científico e Tecnológico) and CAPES (Coordenação de Aperfeiçoamento de Pessoal de Nível Superior).

\section{Author Contributions}

Lucas T. A. da Rosa was responsible for the data curation, investigation, validation-verification, visualization, writing original draft, review and editing; Isabella F. S. Aversa for the investigation, validation-verification; Ellen Raphael for the conceptualization, data curation, project administration, supervision, visualization, writing original draft, review and editing; André S. Polo for the formal analysis, funding acquisition, methodology, supervision, writing review and editing; Alfredo Duarte for the investigation, validation-verification, methodology; Marco A. Schiavon for the formal analysis, funding acquisition, supervision, resources, writing review and editing; Luciano S. Virtuoso for the conceptualization, formal analysis, funding acquisition, investigation, project administration, resources, methodology, visualization, supervision, writing original draft, review and editing. 


\section{References}

1. Samanta, A.; Deng, Z.; Liu, Y.; Yan, H.; Nano Res. 2013, 6, 853.

2. Murray, C. B.; Norris, D. J.; Bawendi, M. G.; J. Am. Chem. Soc. 1993, 115, 8706.

3. Kamat, P. V.; J. Phys. Chem. Lett. 2013, 4, 908.

4. Gao, X.; Li, H.; Sun, W.; Chen, Q.; Tang, F.; J. Phys. Chem. C 2009, 113, 7531.

5. Gaponik, N.; Talapin, D. V.; Rogach, A. L.; Hoppe, K.; Shevchenko, E. V.; Kornowski, A.; Eychmüller, A.; Weller, H.; J. Phys. Chem. B 2002, 106, 7177.

6. Al-Ghamdi, A. A.; Alshehrie, A.; Baell, G.; Optik 2020, 208, 164524.

7. Erathodiyil, N.; Ying, J. Y.; Acc. Chem. Res. 2011, 44, 925.

8. Zhang, H.; Cui, Z.; Wang, Y.; Zhang, K.; Ji, X.; Lü, C.; Yang, B.; Gao, M.; Adv. Mater. 2003, 15, 777.

9. Akbari, M.; Rahimi-Nasrabadi, M.; Pourmasud, S.; EghbaliArani, M.; Banafshe, H. R.; Ahmadi, F.; Ceram. Int. 2020, 46, 9979.

10. Gaponik, N.; Talapin, D. V.; Rogach, A. L.; Eychmüller, A.; Weller, H.; Nano Lett. 2002, 2, 803.

11. Wang, M.; Fu, K.; Lin, J.; J. Semicond. 2011, 32, 053004.

12. Virzbickas, K.; Rimkute, L.; Harvie, A. J.; Critchley, K.; J. Exp. Nanosci. 2017, 12, 94.

13. Singh, S.; Sabri, Y. M.; Jampaiah, D.; Selvakannan, P. R.; Nafady, A.; Kandjani, A. E.; Bhargava, S. K.; Mater. Res. Bull. 2017, 90, 260.

14. Fu, H.; Zunger, A.; Phys. Rev. B 1997, 56, 1496.

15. Wang, L. L.; Jiang, J.; J. Nanoparticle Res. 2011, 13, 1301.

16. Pilla, V.; Munin, E.; J. Nanoparticle Res. 2012, 14, 1147.

17. Lin, W.; Zou, W.; Du, Z.; Li, H.; Zhang, C.; J. Nanoparticle Res. 2013, 15, 1629.

18. Ji, X.; Copenhaver, D.; Sichmeller, C.; Peng, X.; J. Am. Chem. Soc. 2008, 130, 5726.

19. Pradhan, N.; Reifsnyder, D.; Xie, R.; Aldana, J.; Peng, X.; J. Am. Chem. Soc. 2007, 129, 9500.

20. Borchert, H.; Talapin, D. V.; Gaponik, N.; McGinley, C.; Adam, S.; Lobo, A.; Möller, T.; Weller, H.; J. Phys. Chem. B 2003, 107, 9662.

21. CRC Handbook of Chemistry and Physics, Internet Version 2005; Lide, D. R., ed.; CRC Press: Boca Raton, FL, USA, 2005.

22. Trung, T.; Cho, W.; Ha, C. S.; Mater. Lett. 2003, 57, 2746.

23. Lohmann, W.; Fowler, C. F.; Moss Jr., A. J.; Perkins, W. H.; Cell. Mol. Life Sci. 1965, 21, 31.

24. Stan, C. S.; Secula, M. S.; Sibiescu, D.; Electron. Mater. Lett. 2012, 8, 275.
25. Tursunkulov, O.; Allabergenov, B.; Abidov, A.; Jeong, S.; Kim, S.; J. Korean Powder Metall. Inst. 2013, 20, 180.

26. Vale, B. R. C.; Vieira, K. O.; Souza, J. C. L.; Ferrari, J. L.; Schiavon, M. A.; Quim. Nova 2015, 38, 22.

27. Resch-Genger, U.; Rurack, K.; Pure Appl. Chem. 2013, 85, 2005.

28. Veiga, E. T.; Müller, A. V.; Ramos, L. D.; Frin, K. P. M.; Polo, A. S.; Eur. J. Inorg. Chem. 2018, 23, 2680.

29. Silva, F. O.; Viol, L. C. D. S.; Ferreira, D. L.; Alves, J. L. A.; Schiavon, M. A.; Quim. Nova 2010, 33, 1933.

30. Yu, W. W.; Qu, L.; Guo, W.; Peng, X.; Chem. Mater. 2003, 15, 2854.

31. Kalytchuk, S.; Zhovtiuk, O.; Kershaw, S. V.; Zbořil, R.; Rogach, A. L.; Small 2016, 12, 466.

32. Rogach, A. L.; Franzl, T.; Klar, T. A.; Feldmann, J.; Gaponik, N.; Lesnyak, V.; Shavel, A.; Eychmüller, A.; Rakovich, Y. P.; Donegan, J. F.; J. Phys. Chem. C 2007, 111, 14628.

33. Nonoguchi, Y.; Nakashima, T.; Kawai, T.; J. Phys. Chem. C 2008, 112, 19263.

34. Zhang, L.; Zu, Q.; Liu, M.; Kong, L.; Jiao, M.; Mu, H.; Wang, D.; Wang, H.; Chen, J.; Yang, C.; Nanoscale Res. Lett. 2017, $12,222$.

35. Wang, X.; Qu, L.; Zhang, J.; Peng, X.; Xiao, M.; Nano Lett. 2003, 3, 1103 .

36. Califano, M.; ACS Nano 2015, 9, 2960.

37. Pearson, R. G.; J. Am. Chem. Soc. 1963, 85, 3533.

38. Wang, Y.; Liu, S.; J. Chil. Chem. Soc. 2012, 57, 1109.

39. Duan, J.; Song, L.; Zhan, J.; Nano Res. 2009, 2, 61.

40. Lovrić, J.; Cho, S. J.; Winnik, F. M.; Maysinger, D.; Chem. Biol. 2005, 12, 1227.

41. Swayambunathan, V.; Hayes, D.; Schmidt, K. H.; Liao, Y. X.; Meisel, D.; J. Am. Chem. Soc. 1990, 112, 3831.

42. Kini, S.; Kulkarni, S. D.; Ganiga, V.; Nagarakshit, T. K.; Chidangil, S.; Mater. Res. Bull. 2019, 110, 57.

43. Heurtault, B.; Saulnier, P.; Pech, B.; Proust, J. E.; Benoit, J. P.; Biomaterials 2003, 24, 4283.

44. Haldar, D.; Bose, S.; Ghosh, A.; Saha, S. K.; Nanoscale Adv. 2019, 1, 1853.

45. Rajh, T.; Mićić, O. I.; Nozik, A. J.; J. Phys. Chem. C 1993, 97, 11999.

46. Wang, J.; Zhang, J.; Deng, D.; Mater. Lett. 2017, 191, 50.

47. Petrović, M.; Chellappan, V.; Ramakrishna, S.; Sol. Energy 2015, 122, 678 .

Submitted: July 22, 2020

Published online: December 3, 2020 